\title{
EXTENSION AND CONVERGENCE THEOREMS FOR FAMILIES OF NORMAL MAPS IN SEVERAL COMPLEX VARIABLES
}

\author{
JAMES E. JOSEPH AND MYUNG H. KWACK
}

(Communicated by Eric Bedford)

Dedicated to Professor Shoshichi Kobayashi at his retirement

\begin{abstract}
Let $\mathcal{H}(X, Y)(\mathcal{C}(X, Y))$ represent the family of holomorphic (continuous) maps from a complex (topological) space $X$ to a complex (topological) space $Y$, and let $Y^{+}=Y \cup\{\infty\}$ be the Alexandroff one-point compactification of $Y$ if $Y$ is not compact, $Y^{+}=Y$ if $Y$ is compact. We say that $\mathcal{F} \subset \mathcal{H}(X, Y)$ is uniformly normal if $\{f \circ \varphi: f \in \mathcal{F}, \varphi \in \mathcal{H}(M, X)\}$ is relatively compact in $\mathcal{C}\left(M, Y^{+}\right)$(with the compact-open topology) for each complex manifold $M$. We show that normal maps as defined and studied by authors in various settings are, as singleton sets, uniformly normal families, and prove extension and convergence theorems for uniformly normal families. These theorems include (1) extension theorems of big Picard type for such families - defined on complex manifolds having divisors with normal crossings - which encompass results of Järvi, Kiernan, Kobayashi, and Kwack as special cases, and (2) generalizations to such families of an extension-convergence theorem due to Noguchi.
\end{abstract}

\section{INTRODUCTION}

In 1957 Lehto and Virtanen [21] defined a function $f$ meromorphic on the complex unit disk $\mathbf{D}=\{z \in \mathbf{C}:|z|<1\}$ to be normal if $\{f \circ \varphi: \varphi \in \mathcal{A}(\mathbf{D})\}$ is normal in the sense of Montel, where $\mathcal{A}(\mathbf{D})$ is the group of conformal automorphisms of D. Since that time the subject of normal maps has been studied intensively, resulting in an extensive development in the single complex variable context and in generalizations to several complex variables settings (see [6], [8], [10], [25] and lists of references in [4] and [18]).

Let $\mathcal{H}(X, Y)(\mathcal{C}(X, Y))$ represent the family of holomorphic (continuous) maps from a complex (topological) space $X$ to a complex (topological) space $Y$, and let $Y^{+}=Y \cup\{\infty\}$ be the Alexandroff one-point compactification of $Y$ if $Y$ is not compact, $Y^{+}=Y$ if $Y$ is compact. If $\mathcal{F} \subset \mathcal{C}(Y, Z)$ and $\mathcal{G} \subset \mathcal{C}(X, Y)$, we denote $\{f \circ g: g \in \mathcal{G}, f \in \mathcal{F}\}$ by $\mathcal{F} \circ \mathcal{G}$. We say that a family $\mathcal{F}$ of holomorphic maps from a complex space $X$ to a complex space $Y$ is uniformly normal if $\mathcal{F} \circ \mathcal{H}(M, X)$

Received by the editors June 8, 1995.

1991 Mathematics Subject Classification. Primary 32A10, 32C10, 32H20, 32A17; Secondary 54C20, 54C35, 54D35, 54C05.

Key words and phrases. Holomorphic maps, normal maps, uniformly normal families, complex spaces, length function, hyperbolic complex manifolds, hyperbolically imbedded, function spaces, continuous extensions, Picard Theorem, compact-open topology, Ascoli-Arzelà Theorem, onepoint compactification. 
is relatively compact in $\mathcal{C}\left(M, Y^{+}\right)$for each complex manifold $M$, and that $f \in$ $\mathcal{H}(X, Y)$ is a normal map if $\{f\}$ is uniformly normal. The topology used on all function spaces is the compact-open topology. Abate [1] has shown that a complex space $X$ is hyperbolic iff $\mathcal{H}(\mathbf{D}, X)$ is relatively compact in $\mathcal{C}\left(\mathbf{D}, X^{+}\right)$.

After preliminaries in $\S 1$, we offer our main results in $\S 2$, providing generalizations of Picard extension theorems from [11], [14], [15], [16], [19], of the MontelCarathéodory Theorem and of a Noguchi extension-convergence theorem (see [5, p. 300], [22, pp. 20-21] and [23, pp. 39-64]). If $X_{0}, Y_{0}$ are subspaces of the topological spaces $X, Y$ respectively and $\mathcal{F} \subset \mathcal{C}\left(X_{0}, Y_{0}\right)$, then $\mathcal{C}[X, Y ; \mathcal{F}]$ will denote the collection of $g \in \mathcal{C}(X, Y)$ which are extensions of elements of $\mathcal{F}$. In this paper, $X_{0}$ will be dense in $X, Y$ will be Hausdorff and, consequently, each such extension of $f \in \mathcal{C}\left(X_{0}, Y_{0}\right)$ will be unique and will be denoted by $\widetilde{f}$. It will be evident from the context which spaces $X_{0}, Y_{0}, X, Y$ are under consideration. If $X_{0}, Y_{0}$ are complex subspaces of complex spaces $X, Y$ respectively we will write $\mathcal{H}\left[X, Y^{+} ; \mathcal{F}\right]=\mathcal{C}\left[X, Y^{+} ; \mathcal{F}\right]$ if $Y^{+}$is a complex space with $Y$ as a complex subspace. Otherwise, $\mathcal{H}\left[X, Y^{+} ; \mathcal{F}\right]=\mathcal{C}\left[X, Y^{+} ; \mathcal{F}\right] \cap \mathcal{H}(X, Y)$. Let $\mathbf{D}^{*}=\mathbf{D}-\{0\}$, the punctured disk. The notation $\bar{A}$ will represent the closure of the subset $A$ of a topological space.

The results listed in $\left(1^{\circ}\right)-\left(6^{\circ}\right)$ below are established in $\S 2$.

Let $M$ be a complex manifold, let $A$ be a divisor on $M$ with normal crossings, let $\mathcal{F} \subset \mathcal{H}(M-A, Y)$ be uniformly normal and let $\overline{\mathcal{F}}$ be the closure in $\mathcal{C}\left(M-A, Y^{+}\right)$. Then

$\left(1^{\circ}\right)$ Each $f \in \overline{\mathcal{F}}$ extends to $\tilde{f} \in \mathcal{C}\left(M, Y^{+}\right)$.

$\left(2^{\circ}\right) \mathcal{C}\left[M, Y^{+} ; \overline{\mathcal{F}}\right]$ is compact in $\mathcal{C}\left(M, Y^{+}\right)$.

$\left(3^{\circ}\right)$ If $\left\{f_{n}\right\}$ is a sequence in $\overline{\mathcal{F}}$ and $f_{n} \rightarrow f$, then $\widetilde{f}_{n} \rightarrow \widetilde{f}$.

$\left(4^{\circ}\right)$ If $M=\mathbf{D}^{m}$ and $M-A=\left(\mathbf{D}^{*}\right)^{m}, \mathcal{H}\left[M, Y^{+} ; \overline{\mathcal{F}}\right]$ is uniformly normal.

Proposition 1.6 of [12] shows that if $X, Y$ are complex spaces, then $\mathcal{F} \subset \mathcal{H}(X, Y)$ is uniformly normal iff $\mathcal{F} \circ \mathcal{H}(\mathbf{D}, X)$ is uniformly normal. We prove, unexpectedly,

$\left(5^{\circ}\right)$ For complex spaces $X, Y, \mathcal{F} \subset \mathcal{H}(X, Y)$ is uniformly normal iff $\mathcal{F} \circ$ $\mathcal{H}\left(\mathbf{D}^{*}, X\right)$ is uniformly normal.

$\left(6^{\circ}\right)$ A complex subspace $X$ of a complex space $Y$ is hyperbolically imbedded in $Y$ iff there exists a distance function $d$ on $Y$ such that each $f \in \mathcal{H}\left(\mathbf{D}^{*}, X\right)$ is distance decreasing with respect to $k_{\mathbf{D}^{*}}$ and $d$ (i.e. $d(f(x), f(y)) \leq k_{\mathbf{D}^{*}}(x, y)$ for all such $f$ and all $\left.x, y \in \mathbf{D}^{*}\right)$.

The authors would like to express their appreciation to the referee for a careful reading of the paper in its original form, and for suggestions, all of which led to improvements which are reflected in the revision.

\section{Preliminaries}

Let $X$ be a complex manifold. The Kobayashi-Royden differential pseudometric on $X$, the infinitesimal form of the Kobayashi pseudodistance $k_{X}$, will be denoted by $K_{X}$; that is,

$$
K_{X}(p, v)=\inf \left\{r>0: \varphi(0)=p,(d \varphi)_{0}(r e)=v \text { for some } \varphi \in \mathcal{H}(\mathbf{D}, X)\right\},
$$

where $p \in X, v \in T_{p}(X)$, the tangent bundle of $X$ at $p, e$ is the unit vector 1 at $0 \in \mathbf{D}$, and $d \varphi$ is the tangent map induced by $\varphi$ between the tangent spaces of D and $X$ ([20, pp. 88-94]). Recently Kobayashi [17] defined another intrinsic pseudodistance $k_{X, Y}$ on $\bar{X}$ and its infinitesimal form $K_{X, Y}$ when $X$ is a complex 
submanifold of a complex manifold $Y$. The functions $k_{X, Y}$ and $K_{X, Y}$ are defined similarly to the way $k_{X}$ and $K_{X}$ are defined but uses the family

$$
\mathcal{F}_{X, Y}=\left\{f \in \mathcal{H}(\mathbf{D}, Y): f^{-1}(Y-X) \text { is at most a singleton }\right\} .
$$

If no $f \in \mathcal{F}_{X, Y}$ satisfies $f(0)=p$ and $(d f)_{0}(r e)=v$, then $K_{X, Y}(p, v)$ is defined to be $\infty$. It was noted by Kobayashi in [17] that $k_{\mathbf{D}^{*}, \mathbf{D}}=k_{\mathbf{D}}$, and, indeed, he proves in his forthcoming book that $k_{\left(\mathbf{D}^{*}\right)^{m}, \mathbf{D}^{m}}=k_{\mathbf{D}^{m}}$ for each positive integer $m$. A length function on a complex manifold $X$ is a real-valued nonnegative continuous function $E$ defined on the tangent bundle $T(X)$ satisfying (1) $E(v)=0$ iff $v=0$, and (2) $E(a v)=|a| E(v)$ for $a \in \mathbf{C}$ and $v \in T(X)$. If $X$ is a complex manifold and $E$ is a length function on $X$, we denote by $d_{E}$ the distance function generated on $X$ by $E$ [20]. The distance function $d_{E}$ is known to generate the topology on $X$ ([20, pp. 8-10]). If $X$ is a complex hyperbolic manifold and $Y$ is a complex manifold with length function $E$, the norm $|d f|_{E}$ of the tangent map for $f \in \mathcal{H}(X, Y)$ with respect to $E$ is defined by

$$
\begin{aligned}
|d f|_{E} & =\sup \left\{\left|(d f)_{p}\right|_{E}: p \in X\right\} \quad \text { where } \\
\left|(d f)_{p}\right|_{E} & =\sup \left\{E\left((d f)_{p}(v)\right): K_{X}(p, v)=1, v \in T_{p}(X)\right\} .
\end{aligned}
$$

(We use simply $|d f|$ and $\left|(d f)_{p}\right|$ when no confusion may arise.) The pull-back of the length function $E$ by $f$ is defined by $f^{*} E(v)=E(d f(v))$ for $v \in T(X)$. For a complex space $X$ the notions of length function and distance function $d_{E}$ associated with a length function $E$ on $X$ may be defined as for a manifold ([20, pp. 8-10]).

Let $\mathcal{F} \subset \mathcal{C}(X, Y)$; we say that $\mathcal{F}$ is evenly continuous from $p \in X$ to $q \in Y$ if for each $U$ open in $Y$ about $q$, there exist $V, W$ open in $X, Y$ about $p, q$ respectively such that $\{f \in \mathcal{F}: f(p) \in W\} \subset\{f \in \mathcal{F}: f(V) \subset U\}$. If $\mathcal{F}$ is evenly continuous from each $p \in X$ to each $q \in Y$, we say that $\mathcal{F}$ is evenly continuous (from $X$ to $Y$ ) [13]. Let $\mathcal{F}(x)=\{f(x): f \in \mathcal{F}\}$. We will have the occasion to rely on the following topological version of the Ascoli-Arzelà Theorem which is readily derived from a Kelley-Morse theorem (Theorem 7.21 in [13]).

Proposition 1.1. Let $X$ be a locally compact space and let $Y$ be a regular space. Then $\mathcal{F} \subset \mathcal{C}(X, Y)$ is relatively compact in $\mathcal{C}(X, Y)$ iff

(a) $\mathcal{F}$ is evenly continuous, and

(b) $\mathcal{F}(x)$ is relatively compact in $Y$ for each $x \in X$.

While it is immediate that each member of a uniformly normal family is a normal map in that setting, Example 1.3 in [12] exhibits that a family of normal maps might fail to be uniformly normal.

The proof of Proposition 1.2 (proved as part of Proposition 1.6 in [12]) is provided here for the sake of completeness. From this proposition it is not difficult to see that some important classes of complex spaces are defined by uniformly normal families. It is also easy to see from this result that if $Y$ is a relatively compact complex subspace of a complex space, this collection of mappings has been introduced and studied by Zaidenberg [26] and called s-normal families. The proof of Proposition 1.3 is omitted.

Proposition 1.2. If $X, Y$ are complex spaces, then $\mathcal{F} \subset \mathcal{H}(X, Y)$ is uniformly normal iff $\mathcal{F} \circ \mathcal{H}(\mathbf{D}, X)$ is relatively compact in $\mathcal{C}\left(\mathbf{D}, Y^{+}\right)$.

Proof. Necessity. Follows from Definition. 
Sufficiency. If $\mathcal{F}$ is not uniformly normal, there is a complex manifold $M$ such that $\mathcal{F} \circ \mathcal{H}(M, X)$ is not relatively compact in $\mathcal{C}\left(M, Y^{+}\right)$and hence, by Proposition 1.1 , is not evenly continuous; since even continuity is a local property, we may assume that $M=\left\{p \in \mathbf{C}^{m}:\|p\|<1\right\}$ for some $m$ and that $\mathcal{F} \circ \mathcal{H}(M, X)$ is not evenly continuous from $\mathbf{0} \in M$ to $q \in Y^{+}$. Choose sequences $\left\{f_{n}\right\}$ in $\mathcal{F},\left\{p_{n}\right\}$ in $M-\{\mathbf{0}\}$, and $\left\{\varphi_{n}\right\}$ in $\mathcal{H}(M, X)$ such that $\left\|p_{n}\right\| \rightarrow 0, f_{n} \circ \varphi_{n}(0) \rightarrow q$ and $f_{n} \circ \varphi_{n}\left(p_{n}\right) \not \rightarrow q$. Define $\lambda_{n} \in \mathcal{H}(\mathbf{D}, X)$ by $\lambda_{n}(z)=\varphi_{n}\left(z p_{n} /\left\|p_{n}\right\|\right)$; we observe that $f_{n} \circ \lambda_{n}(0) \rightarrow q$ while $f_{n} \circ \lambda_{n}\left(\left\|p_{n}\right\|\right) \nrightarrow q$. From Proposition 1.1, $\mathcal{F} \circ \mathcal{H}(\mathbf{D}, X)$ is not relatively compact in $\mathcal{C}\left(\mathbf{D}, Y^{+}\right)$, so $\mathcal{F} \circ \mathcal{H}(\mathbf{D}, X)$ is not uniformly normal.

Proposition 1.3. Let $(Y, \sigma)$ be a locally compact metric space. Let $X$ be a topological space and let $\rho$ be a pseudometric on $X$ which is continuous on $X \times X$. If each $f \in \mathcal{F} \subset \mathcal{C}(X, Y)$ is distance decreasing with respect to $\rho$ and $\sigma$, then $\mathcal{F}$ is relatively compact in $\mathcal{C}\left(X, Y^{+}\right)$.

Recall that a complex subspace $X$ of a complex space $Y$ is hyperbolically imbedded in $Y$ if for $p, q \in \bar{X}, p \neq q$ there are sets $V, W$ open in $Y$ about $p, q$ respectively such that $k_{X}(V \cap X, W \cap X)>0[14]$.

Example 1.4. Royden showed (Theorem 2 in [24]) that a complex manifold $M$ is hyperbolic iff $\mathcal{H}(\mathbf{D}, M)$ is evenly continuous, and Abate showed (Theorem 1.3 in [1]) that $M$ is hyperbolic iff $\mathcal{H}(\mathbf{D}, M)$ is relatively compact in $\mathcal{C}\left(\mathbf{D}, M^{+}\right)$. Hence $\mathcal{H}(\mathbf{D}, M)$ is a uniformly normal family iff $M$ is hyperbolic.

Example 1.5. It is shown in Theorem 2 in [11] that a complex subspace $X$ of a complex space $Y$ is hyperbolically imbedded in $Y$ iff $\mathcal{H}(\mathbf{D}, X)$ is relatively compact in $\mathcal{C}\left(\mathbf{D}, Y^{+}\right)$, i.e. iff $\mathcal{H}(\mathbf{D}, X)$ is a uniformly normal subfamily of $\mathcal{H}(\mathbf{D}, Y)$. This is a generalization of Kiernan's theorem [14] which deals with the case when $X$ is relatively compact in $Y$.

For $r>0$ let $\mathbf{D}_{r}=\{z \in \mathbf{C}:|z|<r\}$ and $\mathbf{D}_{r}^{*}=\mathbf{D}_{r}-\{0\}$.

Theorem 1.6. Let $M$ be a hyperbolic manifold and let $Y$ be a complex space. Then $\mathcal{F} \subset \mathcal{H}(M, Y)$ is uniformly normal iff there is a length function $E$ on $Y$ such that $|d f|_{E} \leq 1$ for each $f \in \mathcal{F}$.

Proof. Necessity. Clearly $\mathcal{F} \circ \mathcal{H}(\mathbf{D}, M)$ is an evenly continuous subset of $\mathcal{H}(\mathbf{D}, Y)$. We will show first that for each length function $E$ on $Y$ and compact $Q \subset Y$ there exists $c>0$ such that $|d f| \leq c$ on $f^{-1}(Q)$ for each $f \in \mathcal{F}$. If $Q \subset Y$ is compact and fails the stated condition for the length function $E$, we choose sequences $\left\{p_{n}\right\}$, $\left\{f_{n}\right\},\left\{v_{n}\right\}$ and $q \in Q$, such that $p_{n} \in M, f_{n} \in \mathcal{F}, v_{n} \in T_{p_{n}}(M), f_{n}\left(p_{n}\right) \in Q$, $K_{M}\left(p_{n}, v_{n}\right)=1, f_{n}\left(p_{n}\right) \rightarrow q$ and $E\left(\left(d f_{n}\right)_{p_{n}}\left(v_{n}\right)\right)>n$. It follows that $\left|\left(d f_{n}\right)_{p_{n}}\right|$ $\rightarrow \infty$ and we choose a sequence $\left\{\varphi_{n}\right\}$ in $\mathcal{H}(\mathbf{D}, M)$ satisfying $\varphi_{n}(0)=p_{n}$ and $\left|\left(d f_{n} \circ \varphi_{n}\right)_{0}\right| \rightarrow \infty$. Let $V$ be a relatively compact neighborhood of $q$ hyperbolically imbedded in $Y$. Since $\mathcal{F} \circ \mathcal{H}(\mathbf{D}, M)$ is an evenly continuous subset of $\mathcal{H}(\mathbf{D}, Y)$, we choose $0<r<1$ such that $f_{n} \circ \varphi_{n}\left(\mathbf{D}_{r}\right) \subset V$ ultimately; the sequence of restrictions of $\left\{f_{n} \circ \varphi_{n}\right\}$ to $\mathbf{D}_{r}$, which we call again $\left\{f_{n} \circ \varphi_{n}\right\}$, is uniformly normal and is consequently relatively compact in $\mathcal{H}\left(\mathbf{D}_{r}, Y\right)$. Some subsequence of $\left\{f_{n} \circ \varphi_{n}\right\}$ converges to $h \in \mathcal{H}\left(\mathbf{D}_{r}, Y\right)$ contradicting $\left|\left(d f_{n} \circ \varphi_{n}\right)_{0}\right| \rightarrow \infty$. Now, to complete the proof of the necessity, choose sequences $\left\{V_{n}\right\},\left\{c_{n}\right\}$ such that $V_{n}$ is open and relatively compact in $Y, \overline{V_{n}} \subset V_{n+1}, \bigcup_{1}^{\infty} V_{n}=Y, c_{n}>0$ and $|d f|_{E} \leq c_{n}$ on $f^{-1}\left(\overline{V_{n}}\right)$ for each $f \in \mathcal{F}$. Choose a positive continuous function $\mu$ on $Y$ such that 
$\mu(q) c_{n} \leq 1$ on $\overline{V_{n}}$. The length function $H$ on $Y$ defined by $H(v)=\mu(q) E(v)$ for $v \in T_{q}(Y)$ satisfies $|d f|_{H} \leq 1$ for each $f \in \mathcal{F}([20$, p. 34] $)$.

Sufficiency. Each $f \in \mathcal{F} \circ \mathcal{H}(\mathbf{D}, M)$ is distance decreasing with respect to $k_{\mathbf{D}}$ and $d_{E}$ and the desired conclusion follows from Propositions 1.2 and 1.3.

Example 1.7. If $f \in \mathcal{H}\left(\mathbf{D}, \mathbf{P}^{1}(\mathbf{C})\right)$, and $\bar{\Delta} \subset \mathbf{D}$ is a closed disk and $\partial \bar{\Delta}$ denotes the boundary of $\bar{\Delta}$, let $J(f(\bar{\Delta}))$ and $L(f(\bar{\Delta}))$ be respectively the spherical area of $f(\bar{\Delta})$ and spherical length of $f(\partial \bar{\Delta})$. Let $h>0$ and

$$
\mathcal{F}(h)=\left\{f \in \mathcal{H}\left(\mathbf{D}, \mathbf{P}^{1}(\mathbf{C})\right): J(f(\bar{\Delta})) \leq h L(f(\bar{\Delta})) \text { for each closed disk } \bar{\Delta} \subset \mathbf{D}\right\} .
$$

Hayman ([9, p. 164]) showed that $\mathcal{F}(h)$ is invariant relative to $\mathcal{A}(\mathbf{D})$, the group of automorphisms of $\mathbf{D}$, and normal in the sense of Montel. The family $\mathcal{F}(h)$ is uniformly normal.

Example 1.8. Let $M$ be a complex manifold, $r>0$, and $\mathcal{F} \subset \mathcal{H}\left(M, \mathbf{P}^{1}(\mathbf{C})\right)$ be a family of maps such that for each $f \in \mathcal{F}$ three points $a_{f}, b_{f}, c_{f} \in \mathbf{P}^{1}(\mathbf{C})-f(M)$ satisfy $\chi\left(a_{f}, b_{f}\right) \chi\left(c_{f}, b_{f}\right) \chi\left(c_{f}, a_{f}\right) \geq r$ where $\chi$ represents the spherical metric. Carathéodory ([3, p. 202]) showed in this situation that $\mathcal{F} \circ \mathcal{H}(\mathbf{D}, M)$ is normal in the sense of Montel. So $\mathcal{F}$ is uniformly normal.

The normal maps studied in [6], [8], and [18] are all normal maps in our sense.

\section{EXTENSION AND CONVERGENCE THEOREMS}

In this section we offer our main results.

Theorem 2.1. Let $N$ be a hyperbolically imbedded complex submanifold of a complex manifold $M$ and let $Y$ be a complex space. The following are equivalent for $\mathcal{F} \subset \mathcal{H}(N, Y):$

(1) $\mathcal{F}$ is uniformly normal.

(2) If $p \in Y$ and $\left\{g_{n}\right\},\left\{z_{n}\right\}$ are sequences in $\mathcal{F} \circ \mathcal{H}\left(\mathbf{D}^{*}, N\right)$, $\mathbf{D}^{*}$, respectively, such that $z_{n} \rightarrow 0$ and $g_{n}\left(z_{n}\right) \rightarrow p$, then for each neighborhood $U$ of $p$ there is an $r$, $0<r<1$, satisfying $g_{n}\left(\mathbf{D}_{r}^{*}\right) \subset U$ ultimately.

(3) There is a length function $E$ on $Y$ such that $f^{*} E \leq K_{N, M}$ for each $f \in \mathcal{F}$.

Proof. (1) $\Longrightarrow$ (2). From Theorem 1.6 and the fact that $N$ is hyperbolic, there exists a length function $E$ on $Y$ such that each $f \in \mathcal{F} \circ \mathcal{H}\left(\mathbf{D}^{*}, N\right)$ is distance decreasing with respect to $k_{\mathbf{D}^{*}}$ and $d_{E}$. The proof that (2) holds may be completed by arguments similar to those in [15] and $(\mathbf{1}) \Longrightarrow(\mathbf{2})$ of Theorem 1 in [11].

(2) $\Longrightarrow$ (3). We show that for any compact $Q \subset Y$ and length function $E$ on $Y$ there exists $c>0$ such that $c E\left((d f)_{p}(v)\right) \leq 1$ when $f \in \mathcal{F}, f(p) \in Q, v \in T_{p}(N)$ and $K_{N, M}(p, v)=1$. The proof may then be completed as in the proof of the necessity of Theorem 1.6. Suppose $Q \subset Y$ is compact and fails the stated condition for the length function $E$. We choose $q \in Q$ and sequences $\left\{f_{n}\right\},\left\{p_{n}\right\},\left\{v_{n}\right\}$ such that $f_{n} \in \mathcal{F}, f_{n}\left(p_{n}\right) \in Q, v_{n} \in T_{p_{n}}(N), E\left(\left(d f_{n}\right)_{p_{n}}\left(v_{n}\right)\right)>n, K_{N, M}\left(p_{n}, v_{n}\right)=1$, and such that $f_{n}\left(p_{n}\right) \rightarrow q$. We choose sequences $\left\{\varphi_{n}\right\}$ in $\mathcal{F}_{N, M},\left\{r_{n}\right\}$ in (1,2) satisfying $\varphi_{n}(0)=p_{n},\left(d \varphi_{n}\right)_{0}\left(r_{n} e\right)=v_{n}$ and $E\left(\left(d f_{n} \circ \varphi_{n}\right)_{0}\left(r_{n} e\right)\right)>n$. Suppose there exists $r, 0<r<1$, such that a subsequence of the sequence of restrictions of $\left\{f_{n} \circ \varphi_{n}\right\}$ to $\mathbf{D}_{r}$, called again $\left\{f_{n} \circ \varphi_{n}\right\}$, satisfies $f_{n} \circ \varphi_{n} \in \mathcal{F} \circ \mathcal{H}\left(\mathbf{D}_{r}, N\right)$; for such $r$ it follows from (2) that $\mathcal{F} \circ \mathcal{H}\left(\mathbf{D}_{r}, N\right)$ is evenly continuous and since $f_{n} \circ \varphi_{n}(0) \rightarrow q$ we again obtain a contradiction as in the proof of the necessity of Theorem 1.6. Alternatively we choose a sequence $\left\{z_{n}\right\}$ in $\mathbf{D}^{*}$ such that $z_{n} \rightarrow 0$ and $\varphi_{n}\left(z_{n}\right) \in M-N$, and a 
sequence $\left\{\alpha_{n}\right\}$ in $\mathcal{A}(\mathbf{D})$ such that $\alpha_{n}(0)=z_{n}$; let $h_{n}=\varphi_{n} \circ \alpha_{n}$ on $\mathbf{D}^{*}$. Then $h_{n} \in \mathcal{H}\left(\mathbf{D}^{*}, N\right), f_{n} \circ h_{n}\left(\alpha_{n}^{-1}(0)\right) \rightarrow q$ and $\alpha_{n}^{-1}(0) \rightarrow 0$. Let $g_{n}=f_{n} \circ h_{n}$ and let $V$ be a neighborhood of $q$ relatively compact and hyperbolically imbedded in $Y$. There exists $r, 0<r<1$, such that ultimately $g_{n}\left(\mathbf{D}_{r}^{*}\right) \subset V$; so ultimately $g_{n}$ extends to $\widetilde{g}_{n} \in \mathcal{H}(\mathbf{D}, Y)$. From Theorem 2 in [11] there exists a subsequence of $\left\{\widetilde{g}_{n}\right\}$, called again $\left\{\widetilde{g}_{n}\right\}$, satisfying $\widetilde{g}_{n} \rightarrow g \in \mathcal{H}(\mathbf{D}, Y)$, a contradiction since $\left|\left(d \widetilde{g}_{n}\right)_{\alpha_{n}^{-1}(0)}\right|=E\left(\left(d f_{n} \circ \varphi_{n}\right)_{0}(e)\right)$. $N$.

(3) $\Longrightarrow$ (1). This follows easily from Theorem 1.6 since $K_{N, M} \leq K_{N}$ on

Recall that a divisor $A$ on a complex manifold $M$ has normal crossings ([20, p. $58]$ ) if at each point of $A$ there exists a system of complex coordinates $z_{1}, \ldots, z_{m}$ for $M$ such that, locally, $M-A=\left(\mathbf{D}^{*}\right)^{r} \times \mathbf{D}^{s} \quad$ with $r+s=m$.

Lemma 2.2. Let $\mathcal{F} \subset \mathcal{H}\left(\left(\mathbf{D}^{*}\right)^{m}, Y\right)$ be uniformly normal. If $\left\{w_{n}\right\},\left\{f_{n}\right\}$ are sequences in $\left(\mathbf{D}^{*}\right)^{m}, \mathcal{F}$ respectively such that $w_{n} \rightarrow w_{0} \in \mathbf{D}^{m}$ and $f_{n}\left(w_{n}\right) \rightarrow p \in Y$, then for each neighborhood $U$ of $p$ there is a neighborhood $W$ of $w_{0}$ in $\mathbf{D}^{m}$ such that ultimately $f_{n}\left(W \cap\left(\mathbf{D}^{*}\right)^{m}\right) \subset U$.

Proof. The proof is by induction on $m$. Equivalence (2) of Theorem 2.1 establishes the result for $m=1$. Suppose the statement is true for the integer $k$ but not for the integer $k+1$. Let $\mathcal{F} \subset \mathcal{H}\left(\left(\mathbf{D}^{*}\right)^{k+1}, Y\right)$ be uniformly normal, let $\left\{w_{n}\right\},\left\{w_{n}^{\prime}\right\}$ be sequences in $\left(\mathbf{D}^{*}\right)^{k+1}$ such that $w_{n} \rightarrow w_{0} \in \mathbf{D}^{k+1}, w_{n}^{\prime} \rightarrow w_{0}$, and let $\left\{f_{n}\right\}$ be a sequence in $\mathcal{F}$ such that $f_{n}\left(w_{n}\right) \rightarrow p$ while $f_{n}\left(w_{n}^{\prime}\right) \not p$. Let $U, V$ be open relatively compact neighborhoods of $p$ such that $\bar{V} \subset U$ and assume that $f_{n}\left(w_{n}^{\prime}\right) \in Y-U$. Let $w_{n}=\left(s_{n}, t_{n}\right), w_{n}^{\prime}=\left(s_{n}^{\prime}, t_{n}^{\prime}\right)$, and $w_{0}=\left(s_{0}, t_{0}\right)$ where $s_{n}, s_{n}^{\prime}, s_{0} \in\left(\mathbf{D}^{*}\right)^{k}$ and $t_{n}, t_{n}^{\prime}, t_{0} \in \mathbf{D}^{*}$. Let

$$
\begin{aligned}
& \mathcal{F}_{1}=\left\{\varphi_{t} \in \mathcal{H}\left(\left(\mathbf{D}^{*}\right)^{k},\left(\mathbf{D}^{*}\right)^{k+1}\right): t \in \mathbf{D}^{*}, \varphi_{t}(s)=(s, t)\right\} \quad \text { and } \\
& \mathcal{F}_{2}=\left\{\psi_{s} \in \mathcal{H}\left(\mathbf{D}^{*},\left(\mathbf{D}^{*}\right)^{k+1}\right): s \in\left(\mathbf{D}^{*}\right)^{k}, \psi_{s}(t)=(s, t)\right\} .
\end{aligned}
$$

Then $\mathcal{F} \circ \mathcal{F}_{1} \subset \mathcal{H}\left(\left(\mathbf{D}^{*}\right)^{k}, Y\right)$ and $\mathcal{F} \circ \mathcal{F}_{2} \subset \mathcal{H}\left(\mathbf{D}^{*}, Y\right)$ are both uniformly normal families; $\left\{f_{n} \circ \varphi_{t_{n}}\right\}$ is a sequence in $\mathcal{F} \circ \mathcal{F}_{1}, s_{n} \rightarrow s_{0}$ and $f_{n} \circ \varphi_{t_{n}}\left(s_{n}\right) \rightarrow p$. By the induction hypothesis we choose a neighborhood $N_{1}$ of $s_{0}$ such that ultimately $f_{n} \circ \varphi_{t_{n}}\left(N_{1} \cap\left(\mathbf{D}^{*}\right)^{k}\right) \subset V$ and $f_{n} \circ \varphi_{t_{n}}\left(s_{n}^{\prime}\right) \in V$. There exists a subsequence of $\left\{f_{n} \circ \varphi_{t_{n}}\left(s_{n}^{\prime}\right)\right\}$, called again $\left\{f_{n} \circ \varphi_{t_{n}}\left(s_{n}^{\prime}\right)\right\}$, such that $f_{n} \circ \varphi_{t_{n}}\left(s_{n}^{\prime}\right) \rightarrow q \in \bar{V}$; $f_{n} \circ \varphi_{t_{n}}\left(s_{n}^{\prime}\right)=f_{n} \circ \psi_{s_{n}^{\prime}}\left(t_{n}\right) ; t_{n}^{\prime} \rightarrow t_{0}$ and we choose a neighborhood $N_{2}$ of $t_{0}$ in $\mathbf{D}$ such that $f_{n} \circ \psi_{s_{n}^{\prime}}\left(N_{2} \cap \mathbf{D}^{*}\right) \subset U$. Ultimately $f_{n} \circ \psi_{s_{n}^{\prime}}\left(t_{n}^{\prime}\right) \in U$, a contradiction.

If $\left\{A_{n}\right\}$ is a sequence of subsets of a topological space we define the limit superior of the sequence $A_{n}$ to be the collection of elements $x$ of the space with the property that each neighborhood of $x$ intersects $A_{n}$ for infinitely many $n$. We use the notation $\lim \sup A_{n}$ for this set.

Theorem 2.3. Let $M$ be a complex manifold, let $A$ be a divisor on $M$ with normal crossings, let $\mathcal{F} \subset \mathcal{H}(M-A, Y)$ be uniformly normal and let $\overline{\mathcal{F}}$ be the closure in $\mathcal{C}\left(M-A, Y^{+}\right)$. Then

(1) Each $f \in \overline{\mathcal{F}}$ extends to $\tilde{f} \in \mathcal{C}\left(M, Y^{+}\right)$.

(2) $\mathcal{C}\left[M, Y^{+} ; \overline{\mathcal{F}}\right]$ is compact in $\mathcal{C}\left(M, Y^{+}\right)$.

(3) If $\left\{f_{n}\right\}$ is a sequence in $\overline{\mathcal{F}}$ and $f_{n} \rightarrow f$, then $\widetilde{f}_{n} \rightarrow \widetilde{f}$. 
(4) For each sequence $\left\{f_{n}\right\}$ in $\overline{\mathcal{F}}$ there is a subsequence $\left\{f_{n_{k}}\right\}$ of $\left\{f_{n}\right\}$ such that $\limsup f_{n_{k}}^{-1}(P) \cap \limsup f_{n_{k}}^{-1}(Q)=\emptyset$ in the topology of $M$ for each pair $P, Q$ of disjoint subsets of $Y$ with $P$ compact in $Y$ and $Q$ closed in $Y$.

(5) If $M$ is hyperbolic and $K_{M-A, M}=K_{M}$, then $\mathcal{H}\left[M, Y^{+} ; \overline{\mathcal{F}}\right]$ is uniformly normal.

Proof. As for the proofs of (1) and (2), we show first that each $f \in \mathcal{F}$ extends to $\tilde{f} \in \mathcal{C}\left(M, Y^{+}\right)$and that $\mathcal{C}\left[M, Y^{+} ; \mathcal{F}\right]$ is relatively compact in $\mathcal{C}\left(M, Y^{+}\right)$. Since the considerations involved are local in nature, we may assume that $M=\mathbf{D}^{m}$, $\mathcal{F} \subset \mathcal{H}\left(\left(\mathbf{D}^{*}\right)^{m}, Y\right)$, and we show that each $f \in \mathcal{F}$ extends to $\tilde{f} \in \mathcal{C}\left(\mathbf{D}^{m}, Y^{+}\right)$and that $\mathcal{C}\left[(\mathbf{D})^{m}, Y^{+} ; \mathcal{F}\right]$ is evenly continuous from $\mathbf{D}^{m}$ to $Y^{+}$; Lemma 2.2 leads to these conclusions as in (1) $\Longrightarrow(\mathbf{2})$ of Theorem 8 and Corollary 7 in [11]. To finish the proof of (1), if $f \in \overline{\mathcal{F}}$, then there exists a sequence $\left\{f_{n}\right\}$ in $\mathcal{F}$ such that $f_{n} \rightarrow f$. There exists a subsequence $\left\{f_{n_{k}}\right\}$ of $\left\{f_{n}\right\}$ such that $\widetilde{f}_{n_{k}} \rightarrow g \in \mathcal{C}\left(M, Y^{+}\right) ; g=\tilde{f}$ so (1) holds. To establish (2) we show that $\mathcal{C}\left[M, Y^{+} ; \overline{\mathcal{F}}\right]=\overline{\mathcal{C}\left[M, Y^{+} ; \mathcal{F}\right]}$. If $g \in \overline{\mathcal{F}}$, choose a sequence $\left\{f_{n}\right\}$ in $\mathcal{F}$ such that $f_{n} \rightarrow g$. It follows that $\widetilde{f}_{n_{k}} \rightarrow \widetilde{g}$ for some subsequence $\left\{f_{n_{k}}\right\}$ of $\left\{f_{n}\right\}$ and one inclusion is established. For the other inclusion if $\left\{f_{n}\right\}$ is a sequence in $\mathcal{F}$ and $\widetilde{f}_{n} \rightarrow g$, then $f_{n} \rightarrow g$ on $M-A$. To see that (3) holds note that, from (2), each subsequence of $\left\{\widetilde{f}_{n}\right\}$ has a convergent subsequence; and that if $\left\{\widetilde{f}_{n_{k}}\right\}$ is a convergent subsequence, then $\widetilde{f}_{n_{k}} \rightarrow \widetilde{f}$. For the proof of (4) we see from (2) that there is a subsequence $\left\{f_{n_{k}}\right\}$ of $\left\{f_{n}\right\}$ such that $\widetilde{f}_{n_{k}} \rightarrow g \in \mathcal{C}\left(M, Y^{+}\right)$. If $P, Q$ are respectively compact and closed subsets of $Y$ and $x \in \limsup f_{n_{k}}^{-1}(P) \cap \limsup f_{n_{k}}^{-1}(Q)$, then $\widetilde{f}_{n_{k}}(x) \rightarrow g(x)$ and for each $V$ open about $x, f_{n_{k}}(V-A) \cap P \neq \emptyset$ and $f_{n_{k}}(V-A) \cap Q \neq \emptyset$ occur frequently. Hence $g(x) \in \bar{P} \cap \bar{Q}$ in $Y^{+}$. Since $P$ is compact in $Y$ and $Q$ is closed in $Y, g(x) \in P \cap Q$. Finally we prove (5). From Theorem 2.1(3), let $E$ be a length function on $Y$ such that $f^{*} E \leq K_{M-A, M}$ for each $f \in \mathcal{F}$. Let $\widetilde{f} \in \mathcal{H}\left[M, Y^{+} ; \overline{\mathcal{F}}\right]$. There is a sequence $\left\{f_{n}\right\}$ in $\mathcal{F}$ such that $f_{n} \rightarrow f$. It follows that $\widetilde{f}^{*} E \leq K_{M-A, M}=K_{M}$.

Remark 2.4. Theorem 2.3(1), (2), and (3) extend work of the authors [11] to uniformly normal families from complex manifolds having divisors with normal crossings to complex spaces. The results in [11] generalize work of Järvi [10], Kobayashi [16], Kwack [19], Kiernan [15], and Noguchi [22], [23]. Theorem 2.3(5) generalizes a second result in [10]. In particular we have the following corollary.

Corollary 2.5. Let $Y$ be a complex space, and let $M$ and $M-A$ be hyperbolic manifolds as defined in any one of the following four cases;

(1) $M-A=\left(\mathbf{D}^{*}\right)^{n-k} \times \mathbf{D}^{k}$ and $M=\mathbf{D}^{n}$.

(2) $M-A=\left(\mathbf{D}^{*}\right)^{n}$ and $M=\mathbf{D}^{n}$.

(3) $A$ is a closed analytic subset of $M$ of codimension at least 2.

(4) $M$ is $n$-dimensional and $A$ is a closed subset of $M$ with (2n-2)-dimensional Hausdorff measure equal to zero.

Let $\mathcal{F} \subset \mathcal{H}(M-A, Y)$ be uniformly normal, and let $\overline{\mathcal{F}}$ be the closure in $\mathcal{C}\left(M-A, Y^{+}\right)$. Then $\mathcal{H}\left[M, Y^{+} ; \overline{\mathcal{F}}\right]$ is uniformly normal.

Proof. The proof follows from Theorem 2.3(5) since in each case $K_{M}=K_{M-A, M}$ (see [2] for (4)).

Theorem 2.6. The following statements are equivalent for complex spaces $X, Y$ and $\mathcal{F} \subset \mathcal{H}(X, Y)$, where $\overline{\mathcal{F} \circ \mathcal{H}\left(\mathbf{D}^{*}, X\right)}$ is the closure in $\mathcal{C}\left(\mathbf{D}^{*}, Y^{+}\right)$: 
(1) $\mathcal{F}$ is uniformly normal.

(2) $\mathcal{F} \circ \mathcal{H}\left(\mathbf{D}^{*}, X\right)$ is uniformly normal.

(3) $\mathcal{C}\left[\mathbf{D}, Y^{+} ; \mathcal{F} \circ \mathcal{H}\left(\mathbf{D}^{*}, X\right)\right]$ is relatively compact in $\mathcal{C}\left(\mathbf{D}, Y^{+}\right)$.

(4) For each sequence $\left\{f_{n}\right\}$ in $\overline{\mathcal{F} \circ \mathcal{H}\left(\mathbf{D}^{*}, X\right)}$ there is a subsequence $\left\{f_{n_{k}}\right\}$ of $\left\{f_{n}\right\}$ such that $\limsup f_{n_{k}}^{-1}(P) \cap \limsup f_{n_{k}}^{-1}(Q)=\emptyset$ in the topology of $\mathbf{D}$ for each pair of disjoint subsets $P, Q$ of $Y$ with $P$ compact in $Y$ and $Q$ closed in $Y$.

(5) $\mathcal{F}$ satisfies the following three conditions:

(a) $\mathcal{F} \circ \mathcal{H}\left(\mathbf{D}^{*}, X\right)$ is relatively compact in $\mathcal{C}\left(\mathbf{D}^{*}, Y^{+}\right)$,

(b) Each $f \in \overline{\mathcal{F} \circ \mathcal{H}\left(\mathbf{D}^{*}, X\right)}$ extends to $\tilde{f} \in \mathcal{C}\left(\mathbf{D}, Y^{+}\right)$, and

(c) If $\left\{f_{n}\right\}$ is a sequence in $\mathcal{F} \circ \mathcal{H}\left(\mathbf{D}^{*}, X\right)$ such that $f_{n} \rightarrow f$, then $\tilde{f}_{n} \rightarrow \tilde{f}$.

Proof. (1) $\Longrightarrow$ (2). Follows from Proposition 1.2(2).

(2) $\Longrightarrow$ (3). From (2) of Theorem 2.3 and the set inclusion

$\mathcal{H}\left[\mathbf{D}, Y^{+} ; \overline{\mathcal{F} \circ \mathcal{H}\left(\mathbf{D}^{*}, X\right)}\right] \cup \mathcal{C}\left[\mathbf{D}, Y^{+} ; \mathcal{F} \circ \mathcal{H}\left(\mathbf{D}^{*}, X\right)\right] \subset \mathcal{C}\left[\mathbf{D}, Y^{+} ; \overline{\mathcal{F} \circ \mathcal{H}\left(\mathbf{D}^{*}, X\right)}\right]$

(3) $\Longrightarrow(\mathbf{1})$. From the fact that $\mathcal{F} \circ \mathcal{H}(\mathbf{D}, X)$ is a subset of the collection of extensions in $(3)$.

$(2) \Longrightarrow(4)$. From (2) and (4) of Theorem 2.3.

(4) $\Longrightarrow$ (3). We show that $\mathcal{C}\left[\mathbf{D}, Y^{+} ; \mathcal{F} \circ \mathcal{H}\left(\mathbf{D}^{*}, X\right)\right]$ is evenly continuous. If this is not the case choose sequences $\left\{f_{n}\right\}$ in $\mathcal{F} \circ \mathcal{H}\left(\mathbf{D}^{*}, X\right),\left\{v_{n}\right\},\left\{x_{n}\right\}$ in $\mathbf{D}^{*}, x \in \mathbf{D}$, $y \in Y$, and open sets $W_{1}, W_{2}$ in $Y$ about $y$ such that $\bar{W}_{1} \subset W_{2}, \bar{W}_{1}$ compact, $v_{n} \rightarrow x, x_{n} \rightarrow x, f_{n}\left(v_{n}\right) \rightarrow y$, and $f_{n}\left(x_{n}\right) \in Y-W_{2}$. For any subsequence $\left\{f_{n_{k}}\right\}$ of $\left\{f_{n}\right\}$ we have $x \in \limsup f_{n_{k}}^{-1}\left(\bar{W}_{1}\right) \cap \limsup f_{n_{k}}^{-1}\left(Y-W_{2}\right)$ even though $\bar{W}_{1} \cap\left(Y-W_{2}\right)=\emptyset$, so (4) does not hold.

$(2) \Longrightarrow(5)$. Condition (a) follows from definition, condition (b) from (1) of Theorem 2.3 and condition (c) from (3) of Theorem 2.3.

(5) $\Longrightarrow(3)$. Let $\left\{f_{n}\right\}$ be a sequence in $\mathcal{F} \circ \mathcal{H}\left(\mathbf{D}^{*}, X\right)$. By condition (a) there exists a subsequence $\left\{f_{n_{k}}\right\}$ of $\left\{f_{n}\right\}$ such that $f_{n_{k}} \rightarrow f \in \mathcal{C}\left(\mathbf{D}^{*}, Y^{+}\right) ; \widetilde{f}_{n_{k}}, \tilde{f}$ exist for each $k$ by condition (b) and $\widetilde{f}_{n_{k}} \rightarrow \widetilde{f}$ by condition (c).

Kiernan [14] gave further illumination to the concept of hyperbolic imbeddedness by showing that a relatively compact complex subspace $X$ of a complex space $Y$ is hyperbolically imbedded in $Y$ iff there is a length function $E$ on $Y$ such that $f^{*} E \leq K_{\mathbf{D}}$ for each $f \in \mathcal{H}(\mathbf{D}, X)$. Our Theorem 2.7 provides additional insight into the role of hyperbolic imbeddedness in Kobayashi's generalization of the big Picard theorem ([16, Theorem 6.1]). Zaidenberg has provided a number of other criteria for hyperbolic imbeddedness and hyperbolicity in [25].

Theorem 2.7. Let $X$ be a complex subspace of a complex space $Y$. The following statements are equivalent:

(1) $X$ is hyperbolically imbedded in $Y$.

(2) $\mathcal{H}\left(\mathbf{D}^{*}, X\right)$ is a uniformly normal subfamily of $\mathcal{H}\left(\mathbf{D}^{*}, Y\right)$.

(3) There exists a length function $E$ on $Y$ such that each $f \in \mathcal{H}\left(\mathbf{D}^{*}, X\right)$ satisfies $f^{*} E \leq K_{\mathbf{D}^{*}}$.

(4) There exists a distance function $d$ on $Y$ such that each $f \in \mathcal{H}\left(\mathbf{D}^{*}, X\right)$ is distance decreasing with respect to $k_{\mathbf{D}^{*}}$ and $d$.

Proof. (1) $\Longrightarrow$ (2). It is obvious that $\mathcal{H}\left(\mathbf{D}^{*}, X\right) \circ \mathcal{H}\left(\mathbf{D}, \mathbf{D}^{*}\right) \subset \mathcal{H}(\mathbf{D}, X)$ and hence $\mathcal{H}\left(\mathbf{D}^{*}, X\right)$ is a uniformly normal subfamily of $\mathcal{H}\left(\mathbf{D}^{*}, Y\right)$ (see Example 1.5$)$. 
(2) $\Longrightarrow(\mathbf{3})$. Since $\mathbf{D}^{*}$ is hyperbolically imbedded in $\mathbf{D}$, by Theorem $2.1(3)$ there exists a length function $E$ on $Y$ satisfying $f^{*} E \leq K_{\mathbf{D}^{*}, \mathbf{D}}=K_{\mathbf{D}} \leq K_{\mathbf{D}^{*}}$ for each $f \in \mathcal{H}\left(\mathbf{D}^{*}, X\right)$.

(3) $\Longrightarrow(4)$. The distance function induced on $Y$ by the length function $E$ promised in (3) meets the requirements of (4).

(4) $\Longrightarrow(\mathbf{1})$. It follows from Proposition 1.3 that $\mathcal{H}\left(\mathbf{D}^{*}, X\right)$ is relatively compact in $\mathcal{C}\left(\mathbf{D}^{*}, Y^{+}\right)$. Let $\left\{f_{n}\right\}$ be a sequence in $\mathcal{H}(\mathbf{D}, X)$ such that $f_{n} \rightarrow f \in \mathcal{C}\left(\mathbf{D}^{*}, Y^{+}\right)$ on $\mathbf{D}^{*}$. We show that $f$ extends to $\tilde{f} \in \mathcal{C}\left(\mathbf{D}, Y^{+}\right)$and that $f_{n} \rightarrow \tilde{f}$ on $\mathbf{D}$. This will complete the proof of $(\mathbf{4}) \Longrightarrow(\mathbf{1})$ in view of the result referred to in Example 1.5. If for each compact $Q \subset Y$ there exists a neighborhood $V$ of 0 in $\mathbf{D}$ ultimately satisying $f_{n}(V) \cap Q=\emptyset, f$ may be extended to $\widetilde{f} \in \mathcal{C}\left(\mathbf{D}, Y^{+}\right)$by defining $\widetilde{f}(0)=\infty$, and $f_{n} \rightarrow \widetilde{f}$ on $\mathbf{D}$. Otherwise, choose a subsequence of $\left\{f_{n}\right\}$, called again $\left\{f_{n}\right\}$, a sequence $\left\{z_{n}\right\}$ in $\mathbf{D}^{*}$, and $p \in Y$ such that $\left|z_{n}\right| \downarrow 0, \quad f_{n}\left(z_{n}\right) \rightarrow p$. If $r_{n} \downarrow 0$, the hyperbolic length of $\sigma_{r_{n}}=\left\{z \in \mathbf{D}:|z|=r_{n}\right\}$ in $\mathbf{D}^{*}$ converges to 0 ; it follows from (4) and winding number arguments modeled after those of Grauert and Reckziegel ([7, p. 120]) and found in [11] and [15], that $f_{n}(0) \rightarrow p, \quad f_{n}\left(z_{n}^{\prime}\right) \rightarrow p$ for any sequence $\left\{z_{n}^{\prime}\right\}$ for which $z_{n}^{\prime} \rightarrow 0$. Hence $f$ may be extended to $\widetilde{f} \in \mathcal{C}\left(\mathbf{D}, Y^{+}\right)$by defining $\widetilde{f}(0)=p$, and $f_{n} \rightarrow \tilde{f}$ on $\mathbf{D}$.

Remark 2.8. The equivalence $(\mathbf{1}) \Longleftrightarrow(\mathbf{3})$ in Theorem 2.7 shows surprisingly that the statement obtained by dropping the relative compactness on $X$ and replacing $\mathbf{D}$ by $\mathbf{D}^{*}$ in Kiernan's result is valid.

Remark 2.9. Let $X$ be a complex subspace of a complex space $Y$. Kwack's generalization of the big Picard theorem establishes that $f \in \mathcal{H}\left(\mathbf{D}^{*}, X\right)$ is extendable to $\tilde{f} \in \mathcal{H}(\mathbf{D}, Y)$ if (1) There exists a distance function $d$ on $Y$ such that $f$ is distance decreasing with respect to $k_{\mathbf{D}^{*}}$ and $d$, and (2) There exists a sequence $\left\{z_{n}\right\}$ in $\mathbf{D}^{*}$ and a $p \in Y$ such that $z_{n} \rightarrow 0$ and $f\left(z_{n}\right) \rightarrow p$ (Theorem 3 in [19]). We observe from $(\mathbf{1}) \Longleftrightarrow(\mathbf{3})$ in Theorem 2.7 that under the hypothesis of Kobayashi's generalization [16] of Kwack's theorem, all $f \in \mathcal{H}\left(\mathbf{D}^{*}, X\right)$ satisfy conditions (1) and (2).

Remark 2.10. The equivalence $(\mathbf{1}) \Longleftrightarrow(\mathbf{2})$ of Theorem 2.7 establishes that a complex space $X$ is hyperbolic iff $\mathcal{H}\left(\mathbf{D}^{*}, X\right)$ is a uniformly normal subfamily of $\mathcal{H}\left(\mathbf{D}^{*}, X\right)$ (compare with Abate's characterization cited in Example 1.4).

\section{REFERENCES}

1. M. Abate, A characterization of hyperbolic manifolds, Proc. Amer. Math. Soc. 117 (1993), 789-793. MR 93d:32037

2. L. A. Campbell, A. Howard and T. Ochiai, Moving holomorphic disks off analytic subsets, Proc. Amer. Math. Soc. 60 (1976), 106-108. MR 54:13143

3. C. Carathéodory, Theory of Functions, vol. II, Chelsea, New York, 1954. MR 16:346c

4. E. F. Collingwood and A. J. Lohwater, The Theory of Cluster Sets, Cambridge University Press, London, 1966. MR 38:325

5. J. B. Conway, Functions of One Complex Variable, Springer-Verlag, New York, 1978. MR 80c: 30003

6. K. Funahashi, Normal holomorphic mappings and classical theorems of function theory, Nagoya Math. J. 94 (1984), 89-104. MR 86b:32029

7. H. Grauert and H. Reckziegel, Hermitesche Metriken und normale Familien holomorpher Abbildunger, Math. Z. 89 (1965), 108-125. MR 33:2827

8. K. T. Hahn, Non-Tangential limit theorems for normal mappings, Pac. J. Math. 135 (1988), 57-64. MR 89i:32010 
9. W. K. Hayman, Meromorphic Functions, Oxford Univ. Press, Oxford, 1964. MR 29:1337

10. P. Järvi, An Extension theorem for normal functions in several variables, Proc. Amer. Math. Soc. 103 (1988), 1171-1174. MR 89h:32048

11. J. E. Joseph and M. H. Kwack, Hyperbolic imbedding and spaces of continuous extensions of holomorphic maps, Jour. Geom. Analysis 4 (3) (1994), 361-378. MR 95g:32027

12. Some classical theorems and families of normal maps in several complex variables, Complex Variables 29 (1996), 343-362. CMP 96:13

13. J. L. Kelley, General Topology, Van Nostrand, Princeton, N.J., 1955. MR 16:1136c

14. P. Kiernan, Hyperbolically imbedded spaces and the big Picard theorem, Math. Ann. 204 (1973), 203-209. MR 51:8476

15. , Extensions of holomorphic maps, Trans. Amer. Math. Soc. 172 (1972), 347-355. MR 47:7066

16. S. Kobayashi, Hyperbolic Manifolds and Holomorphic Mappings, Marcel Dekker, New York, 1970. MR 43:3503

17. _ Relative intrinsic distance and hyperbolic imbedding, Symposia Mathematica, Proceedings of "Recent Advances in Differential Geometry", Pisa, 1993, 36 (to appear).

18. S. G. Krantz, Geometric Analysis and Function Spaces, CBMS, vol. 81, Amer. Math Soc., Providence, RI., 1993. MR 94g:32001

19. M. H. Kwack, Generalization of the big Picard theorem, Ann. of Math. 90 (2) (1969), 9-22. MR 39:4445

20. S. Lang, Introduction to Complex Hyperbolic Spaces, Springer -Verlag, N.Y., 1987. MR 88f:32065

21. O. Lehto and K. I. Virtanen, Boundary behaviour and normal meromorphic functions, Acta Math. 97 (1957), 47-65.

22. J. Noguchi, Hyperbolic fiber spaces and Mordell's conjecture over function fields, Publ. Research Institute Math. Sciences Kyoto University 21 (1) (1985), 27-46. MR 86k:32022

23. Moduli spaces of holomorphic mappings into hyperbolically imbedded complex spaces and locally symmetric spaces, Invent. Math. 93 (1988), 15-34. MR 89j:32031

24. H. Royden, Remarks on the Kobayashi metric, Proc. Maryland Conference on Several Complex Variables, Lecture Notes, vol. 185, Springer-Verlag, Berlin, 1971, pp. 125-137. MR 46:3826

25. M. G. Zaidenberg, Picard's theorem and hyperbolicity, Siberian Math. J. 24 (1983), 858-867. MR 85e: 32033

26. _ Schottky-Landau growth estimates for s-normal families of holomorphic mappings, Math. Ann. 293 (1992), 123-141. MR 93c:32037

Department of Mathematics, Howard University, Washington, D. C. 20059

E-mail address: jjoseph@scs.howard.edu 